\title{
Streaming Potential Measurements on the Binary Mixture Triethylamine-Water Near the Demixing Phase Transition
}

\author{
Luong Duy Thanh ${ }^{1}{ }^{1}$ and Rudolf Sprik ${ }^{2}{ }^{2}$ \\ ${ }^{1}$ Thuyloi University, 175 Tay Son, Dong Da, Ha Noi, Vietnam \\ ${ }^{2}$ Van der Waals-Zeeman Institute, University of Amsterdam, 1098XH Amsterdam, Netherlands \\ Correspondence should be addressed to Luong Duy Thanh; luongduythanh2003@yahoo.com
}

Received 13 January 2019; Accepted 20 March 2019; Published 22 April 2019

Guest Editor: Jan Vinogradov

Copyright (c) 2019 Luong Duy Thanh and Rudolf Sprik. This is an open access article distributed under the Creative Commons Attribution License, which permits unrestricted use, distribution, and reproduction in any medium, provided the original work is properly cited.

\begin{abstract}
Large density fluctuations developing near the phase transition point of the binary mixture affect physical parameters directly related to the electrokinetic coupling coefficient. Here the first electrokinetic measurements for a porous rock sample are carried out with a critical binary mixture of triethylamine-water, especially around the phase transition point. From the measured streaming potential coefficient, the zeta potential is obtained for the critical composition. The results show that there is no anomaly in the streaming potential coefficient as the temperature approaches the demixing temperature. It is also seen that the streaming potential coefficient and the zeta potential in magnitude decreases with increasing temperature. This observation is opposite to what has been observed in literature. It means that the properties of the electric double layer for the mixtures are different from those for aqueous electrolytes. Additionally, the zeta potential for the critical composition is predicted to fluctuate around the critical point.
\end{abstract}

\section{Introduction}

Electrokinetic phenomena are induced by the relative motion between a fluid and a solid surface and are directly related to the existence of an electric double layer between the fluid and the solid grain surface. Electrokinetic phenomena consist of several different effects such as streaming potential, electroosmosis, and electrophoresis. Of electrokinetic phenomena, streaming potential effect plays an important role in geophysical applications. For example, streaming potential could be used to map subsurface flow and detect subsurface flow patterns in oil reservoirs (e.g., $[1,2]$ ), geothermal areas, and volcanoes (e.g., [3-5]). It has also been proposed to use the streaming potential monitoring to detect at distance the propagation of a water front in a reservoir [6]. Monitoring of streaming potential anomalies has been proposed as a means of predicting earthquakes (e.g., $[7,8]$ ). Most experimental measurements of streaming potential in porous media have been performed with electrolyte aqueous solutions (e.g.) [913]). However, in reality the liquids found underground can be a complicated mixture of water, crude oil, and gas. It should be noted that at a certain condition (high temperature and high pressure), oil and water can mix together. Hence, studying electrokinetics or streaming potential with liquid mixtures may be relevant in oil recovery. The effect of the compositions on the electrokinetic phenomena happening in some liquid mixtures has been reported in the literature. For example, the influences of the compositions on the electrophoresis $[14,15]$, on the electroosmosis $[16,17]$, and on the streaming potential [16] have been carried out with liquid mixtures such as methanol-water, ethanol-water, 2-propanolwater, 1-propanol-water, methanol-acetone-water, alcoholwater, and acetone-water. Additionally, the impact of reactive mixing of fluids with different chemical compositions in the subsurface on the resulting effective electrical conductivity that is observed to significantly change over several orders of magnitudes in comparison with the background solution is proposed in [18]. Consequently, it is inferred from those studies that the compositions of the mixtures affect the electrokinetic coupling coefficients. The changes of the electrokinetic coupling coefficients have been explained by both the change of the characteristic parameters of the 


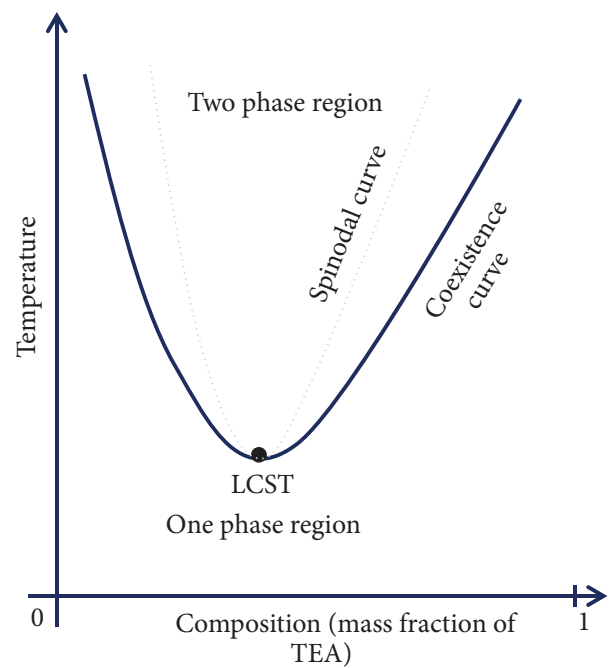

Figure 1: A phase diagram for a case of lower critical solution temperature (LCST) [24].

mixtures (the electrical conductivity, viscosity, density, and dielectric constant) and the variation of the zeta potential with compositions.

However, to the best of our knowledge the streaming potential measurement in binary mixtures near a demixing phase transition in a porous system has not yet been studied. The transition from the one phase solution to the two phase solution passes through an intermediate state called the spinodal decomposition, which is characterized by a solution that strongly scatters light and becomes opalescent. The reason is that close to the phase separation temperature, the scattering efficiency becomes much larger because not only the density contrast increases but also the length scale of the fluctuating regions grows and starts to match the wavelength of the scattered light $[19,20]$. The temperature, corresponding to the state of maximum scattering, is plotted as a function of mass fraction, yielding a phase diagram as shown in Figure 1, for example, for the case of the lower critical solution temperature (LCST). At temperatures below LCST, the system is completely miscible in all proportions, whereas above LCST partial liquid miscibility occurs. The point (LCST) at which the curve has a zero slope is the critical point that corresponds to the critical composition and critical temperature [19]. As shown in Figure 1, the components of a mixture are miscible for all composition below the LCST point.

The motivation for this work is that large density fluctuations developing near the critical point of the binary mixtures affect physical parameters directly related to streaming potential coefficient (the electrokinetic coupling coefficient in general) such as viscosity, electrical conductivity, and dielectric constant [21-23]. From those observations, we investigate how streaming potential coefficient and the zeta potential, one of the most important parameters in electrokinetics, behave near the demixing phase transition.
The streaming potential measurements in an artificial ceramic porous sample are performed with a triethylaminewater binary mixture (TEA-W). This mixture is a typical partially miscible system that has a lower critical solution temperature near room temperature at atmospheric pressure. The critical composition of the mixture is $32.1 \%$ of TEA mass fraction and critical temperature ranges from $18.24^{\circ} \mathrm{C}$ to $18.40^{\circ} \mathrm{C}[21,23]$. The experimental measurements are carried out in the single phase solution. Three different mass fractions of TEA $(20 \%, 32.1 \%$, and $70 \%)$ are used for the measurements. Based on the measured streaming potential coefficient with the knowledge of electrical conductivity, viscosity, and dielectric constant, the zeta potential is obtained for the critical composition. The results show that there is no anomaly in the streaming potential coefficient as the temperature approaches the demixing temperature for all three mass fractions of TEA. It is also seen that the streaming potential coefficient and the zeta potential in magnitude decrease with increasing temperature. This observation is opposite to what has been observed in literature for aqueous electrolytes. It means that the properties of the electric double layer for the TEA-W mixtures are different from those for aqueous electrolytes. As a consequence of the fluctuations of the viscosity and the dielectric constant of the binary mixture at the critical composition, the zeta potential is predicted to fluctuate around the critical point.

This work has four sections. In the first we briefly describe the theoretical background of streaming potential. In the second we present the experimental measurements. The third section contains the experimental results. Conclusions are provided in the final section.

\section{Theoretical Background of Streaming Potential}

Surfaces of the minerals of porous media are generally electrically charged, creating an electric double layer (EDL) containing an excess of charge that counterbalances the charge deficiency of the mineral surface (e.g., [28]). Figure 2 shows structure of the EDL: a Stern layer that contains only counterions coating the mineral with a very limited thickness and a diffuse layer that contains both counterions and coions but with a net excess charge. The shear plane that can be approximated as the limit between the Stern layer and diffuse layer separates the mobile and immobile part of the water molecules when subjected to a fluid pressure difference. The electrical potential at the shear plane is called the zeta potential $(\zeta)$. The zeta potential is a complicated function of many parameters such as mineral composition of porous media, ionic species present in the fluid, the $\mathrm{pH}$ of fluid, fluid electrical conductivity, and temperature (see $[29,30]$ for more details). In the bulk liquid, the numbers of cations and anions are equal so that it is electrically neutral. Most reservoir rocks have a negative surface charge and a negative zeta potential when they are in contact with ground water.

The streaming current is generated by the relative movement of the diffuse layer with the solid surface due to a fluid pressure difference over the capillary (a porous medium 


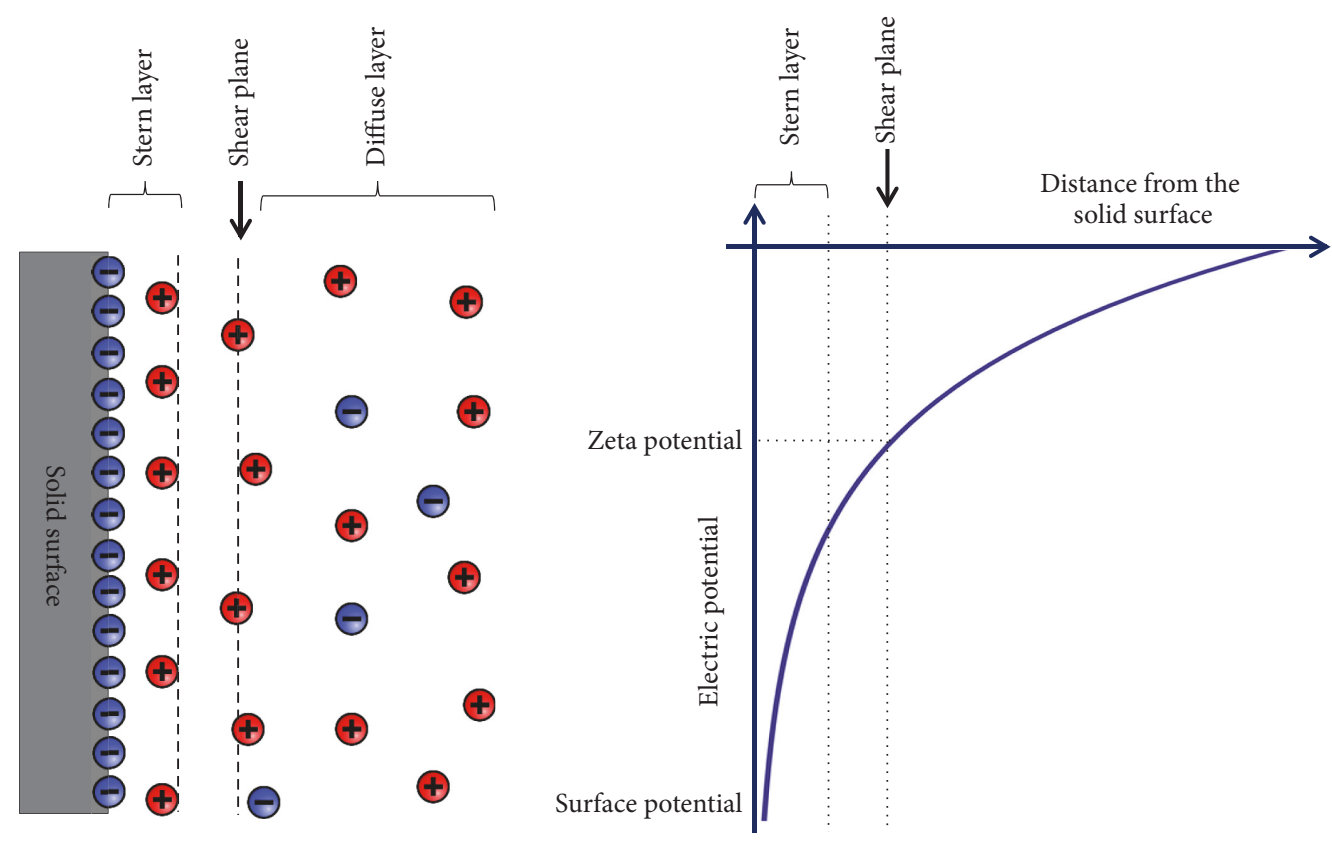

FIGURE 2: Stern model (see [25-27] for more details) for the charge and electric potential distribution in the electric double layer at a solidliquid interface. In this figure, the solid surface is negatively charged and the mobile counter-ions in the diffuse layer are positively charged.

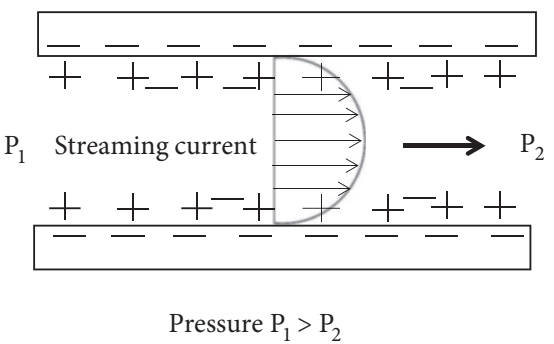

FIGURE 3: Development of streaming potential when an electrolyte is pumped through a capillary (a porous medium is made of an array of capillaries).

can be considered as a bundle of capillaries). This streaming current is balanced by a conduction current, leading to the streaming potential (Figure 3). In a fluid saturated porous medium, the electric current density and the fluid flux are coupled, so fluids moving through porous media generate a streaming potential [31] (Figure 4). The streaming potential increases linearly with the fluid pressure difference that drives the fluid flow, provided that the flow remains laminar [11]. The streaming potential coefficient (SPC) is defined when the total current density is zero as (e.g., $[27,32])$

$$
C_{S}=\frac{\Delta V}{\Delta P}=\frac{\epsilon_{r} \epsilon_{0} \zeta}{\eta \sigma_{e f f}}
$$

where $\Delta V$ is the streaming potential, $\Delta P$ is the fluid pressure difference, $\epsilon_{r}$ is the relative permittivity of the fluid, $\epsilon_{0}$ is the dielectric permittivity in vacuum, $\eta$ is the dynamic viscosity of the fluid, $\sigma_{e f f}$ is the effective conductivity, and $\zeta$ is the zeta potential. The effective conductivity includes the fluid conductivity and the surface conductivity. The SPC can also be written as $[33,34]$

$$
C_{S}=\frac{\epsilon_{r} \epsilon_{0} \zeta}{\eta F \sigma_{r}}
$$

where $\sigma_{r}$ is the electrical conductivity of the sample saturated by a fluid with a conductivity $\sigma_{f}$ and $F$ is the formation factor. The electrical conductivity of the sample can possibly include surface conductivity. If the fluid conductivity is much higher than the surface conductivity, one has $\sigma_{e f f}=\sigma_{f}$. Equation (1) is rewritten as

$$
C_{S}=\frac{\epsilon_{r} \epsilon_{0} \zeta}{\eta \sigma_{f}} .
$$

\section{Experiments}

The triethylamine (TEA) is purchased from VWR (Merck). The purity is stated to be $99.95 \%$. The TEA is used without further purification. The water used in this work is deionized water. All mixtures are prepared from weighed amounts of the pure components. Besides the critical composition of $32.1 \%$ mass fraction, two other mass fractions of $20 \%$ and $70 \%$ are also used in our work. The porous sample is a cylindrical artificial rock made of alumina (72\%) and fused silica (28\%) from HP Technical Ceramics (55 mm in length and $25 \mathrm{~mm}$ in diameter). The microstructure properties of the sample have been reported in $[35,36]$ for the sample labeled DP217: the permeability of $370 \mathrm{mD}$, the porosity of $45.4 \%$, the solid density of $3652 \mathrm{~kg} / \mathrm{m}^{3}$, and the formation factor of 4.5 .

The experimental setup for the streaming potential measurement is similar to that described in [35-37] (see Figure 5). To control temperature of the mixture, the core holder 


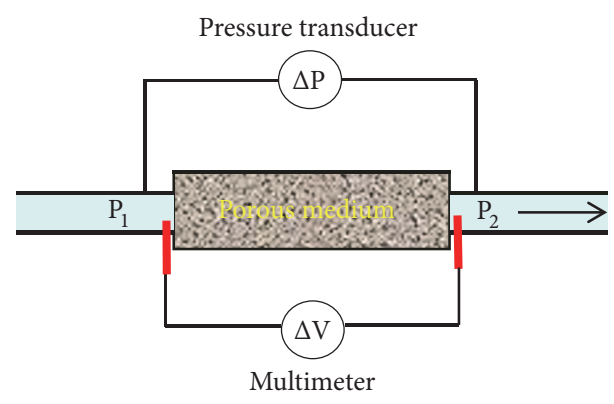

FIGURE 4: Schematic illustration of streaming potential measurement when an electrolyte is pumped through a porous medium. $\Delta \mathrm{V}=\mathrm{V}_{\mathrm{P} 1}-\mathrm{V}_{\mathrm{P} 2}$ is the streaming potential and $\Delta \mathrm{P}=\mathrm{P}_{\mathrm{P} 1}-\mathrm{P}_{\mathrm{P} 2}$ is the fluid pressure difference.

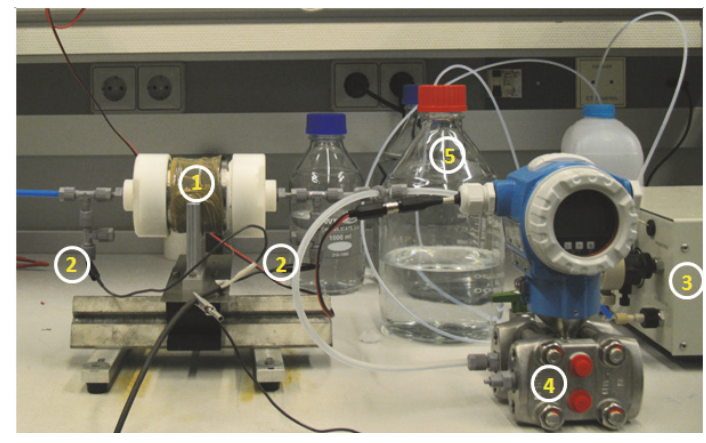

FIGURE 5: Experimental setup for streaming potential measurements described in [35, 36]. (1) Core holder; (2) Ag/ $\mathrm{AgCl}$ electrodes; (3) pump; (4) pressure transducer; (5) solution container.

(Figure 6) and the solution container, in which TEA and water have been mixed together, are immersed in a thermally stabilized water bath (Thermo Fisher Scientific SC150 and A25). The sample is surrounded by a conical Teflon sleeve inserted into a Teflon cell as shown in Figure 6. By tightly twisting the chambers over the Teflon cell, the fluid can be prevented from flowing along the interface of the sample. The chambers on both sides are made of perspex and are transparent to check whether there is a phase transition around the critical temperature or not (the solution becomes opalescent). Rubber is not used in the experimental setup because it is attacked by TEA. A whole experimental setup is placed in a laboratory fume hood to prevent the terrible smell of triethylamine.

The solution is circulated through the sample until the electrical conductivity of the mixture reaches a stable value. The pressure differences across the sample are created by the high pressure pump (LabHut Series III- Pump) and measured by the pressure transducer (Endress and Hauser Deltabar $S$ PMD75). Electrical potentials across the sample are then measured by a high input impedance multimeter (Keithley Model 2700) using two $\mathrm{Ag} / \mathrm{AgCl}$ wire electrodes. The input resistance of the multimeter is larger than $10 \mathrm{G} \Omega$. The resistance of the samples is usually smaller than $250 \mathrm{k} \Omega$, which is low compared to input resistance of the multimeter, therefore allowing accurate measurements of electric potentials. The

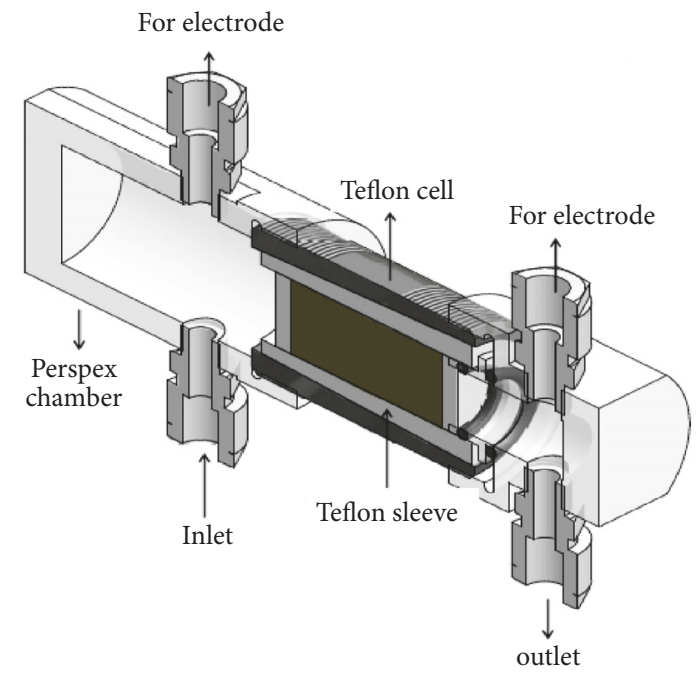

FIgURE 6: The core holder.

LabVIEW program averages the value of electrical potentials. The $\mathrm{Ag} / \mathrm{AgCl}$ electrodes are made of electrolysis of a wire of clean silver from VWR company in $0.1 \mathrm{M} \mathrm{NaCl}$ with an electrical current of $20 \mathrm{~mA}$ and in 2 minutes (for more details, see [27]). The electrodes are covered by heat shrinkable Teflon tubes, and their tips are put in the vicinity of the end faces of the sample but not within the liquid circulation to avoid the electrical noise from liquid movement around the electrodes [33].

\section{Results and Discussion}

The experimental measurements are carried out in the single phase solution. The solution container is firstly cooled down to $10^{\circ} \mathrm{C}$ and stirred; a single phase solution is formed in the container. The container is then slowly heated up until the entire volume of the solutions becomes white and the opalescence is easily visible due to strong light scattering. The critical temperature for the $32.1 \%$ composition is visually identified to be $20.2^{\circ} \mathrm{C}$ in our measurements when the opalescence is well developed in the glass container that can be seen through. The critical temperature is shifted to a higher value compared to the known critical temperature $T_{c}=18.4^{\circ} \mathrm{C}$ [21] or $18.242^{\circ} \mathrm{C}<T_{c}<18.253^{\circ} \mathrm{C}$ [23]. The reasons for this discrepancy are likely the impurities (e.g., $\mathrm{CO}_{2}, \mathrm{H}_{2} \mathrm{O}$ ) of the mixtures, the dissolution of mineral substances of the sample, and the ability of the strongly basic triethylamine to attack glass as mentioned in [19]. Using the same procedure to obtain the demixing temperatures $\left(\mathrm{T}_{\text {demix }}\right)$ for two other mass fractions, we obtain the $\mathrm{T}_{\text {demix }}$ of $21^{\circ} \mathrm{C}$ and $19^{\circ} \mathrm{C}$ for the mass fractions of TEA of $20 \%$ and $70 \%$, respectively.

The way used to collect the SPC is similar to that described in $[35,36]$. Figure 7 shows an example of the electric potential as a function the pressure difference across the sample for the $32.1 \%$ mass fraction at $10^{\circ} \mathrm{C}$. From that graph, the SPC as the slope of the straight line is obtained. To see how the SPC and the zeta potential behave around the phase transition point, temperature is scanned from $10^{\circ} \mathrm{C}$ 


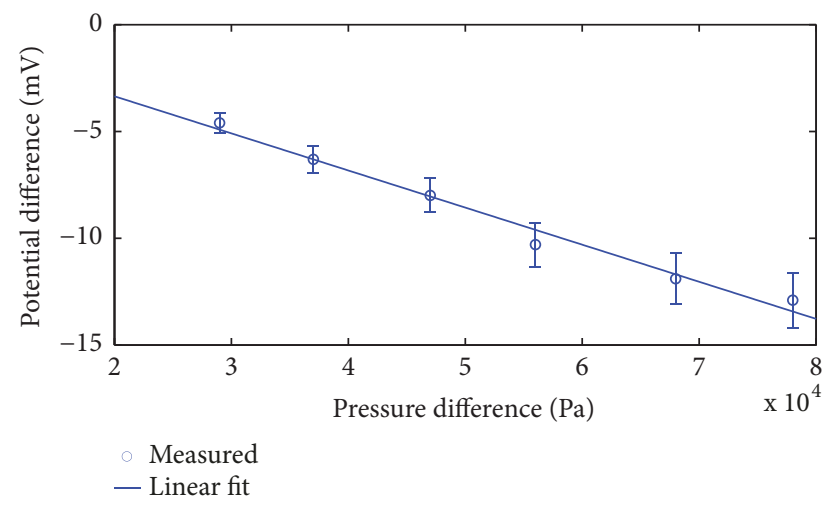

FIGURE 7: Streaming potential as a function of pressure difference for the $32.1 \%$ mass fraction at $10^{\circ} \mathrm{C}$. The symbols are the measured data. The solid line is the linear fit.

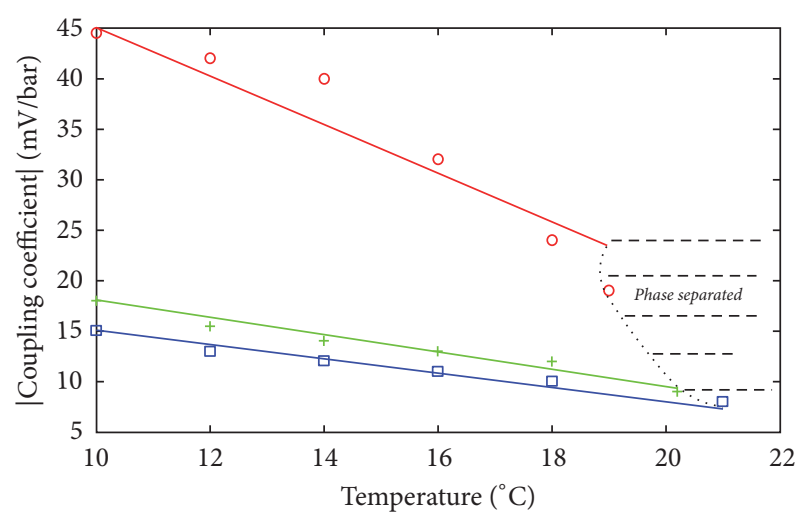

- Measured for $70 \%$

+ Measured for $32.1 \%$

๑ Measured for $20 \%$

FIGURE 8: Streaming potential coefficient as a function of temperature at 3 different mass fractions of TEA (20\%, 32.1\%, and $70 \%$ mass). The symbols are the measured data. The solid lines are the fitting ones.

to $\mathrm{T}_{\text {demix }}\left(21^{\circ} \mathrm{C}, 20.2^{\circ}\right.$, and $19^{\circ} \mathrm{C}$ for the mass fractions of $20 \%, 32.1 \%$, and $70 \%$, respectively). It should be noted that if temperature was raised higher than the $\mathrm{T}_{\text {demix }}$, the solution pumped through the sample would be either TEA or water rather than the mixture due to the phase separation.

The SPC as a function of temperature in one phase solution for three different mass fractions of TEA is shown in Figure 8. It should be noted that the separation between mixed and demixed mixtures is roughly indicated by a faint line for clarity in Figure 8. It is seen that the SPC in magnitude decreases with increasing temperature. To remove the electric potential disturbance of the solid particle or noise from the setup itself, the variation of the SPC with temperature for $\mathrm{NaCl}$ electrolyte only at $5 \cdot 10^{-3} \mathrm{M}$ that has been reported in [35] for the same sample is indicated in Figure 9. It is seen that the SPC in magnitude increases with increasing temperature for $\mathrm{NaCl}$ electrolyte only, which is in good agreement with those normally observed for aqueous electrolyte-rock systems (e.g., $[26,38])$. Consequently, the

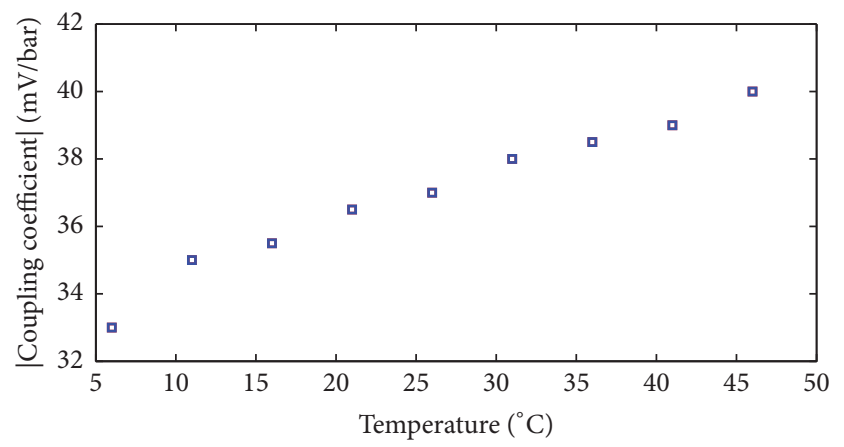

FIGURE 9: The variation of the streaming potential coefficient with temperature for $\mathrm{NaCl}$ electrolyte only at $5.10^{-3} \mathrm{M}$ obtained from [35].

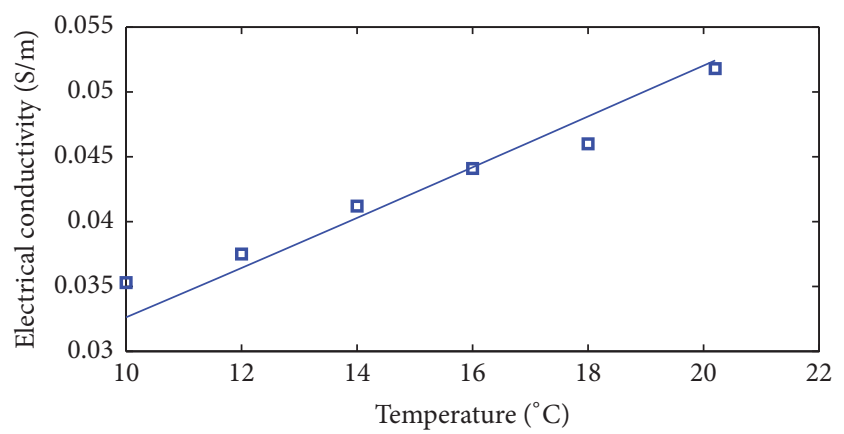

FIGURE 10: Electrical conductivity of the mixture as a function of temperature at the critical composition (32.1\%). The square symbols are the measured data and the solid line is the fitting one from (4) with the parameters $\mathrm{A}=0.37 \mathrm{mS} / \mathrm{cm}, \mathrm{B}=102.0 \mathrm{~K}^{-1}$, and $T_{g}=241.0 \mathrm{~K}$.

measured result in this work is opposite to what has been reported for the same sample with $\mathrm{NaCl}$ electrolyte only. It suggests that there is a variation in the properties of the EDL between using the TEA-W mixture and using aqueous electrolytes. The result also shows that there is no anomaly of the SPC when temperature approaches the $\mathrm{T}_{\text {demix }}$ for three mass fractions of TEA.

In order to calculate the zeta potential from the measured SPC at the critical composition, for example, one needs to know the electrical conductivity, viscosity, and dielectric constant of the mixture. Due to the availability of the viscosity and dielectric constant of the mixture in literature, the zeta potential is only obtained for the critical composition (32.1\%). The electrical conductivity of the mixture as a function of temperature is directly measured by the conductivity meter (Consort C861) and is shown in Figure 10. It is shown that the electrical conductivity of the binary mixture increases with increasing temperature as expected in [39] in which the relationship between electrical conductivity and temperature is given by Vogel-Fulcher-Tammann (VFT) equation

$$
\sigma=A \exp \left(-\frac{B}{T-T_{g}}\right),
$$

where $\mathrm{A}, \mathrm{B}$, and $T_{g}$ are fitting parameters. By fitting the experimental data to (4) as shown in Figure 10 (the solid 


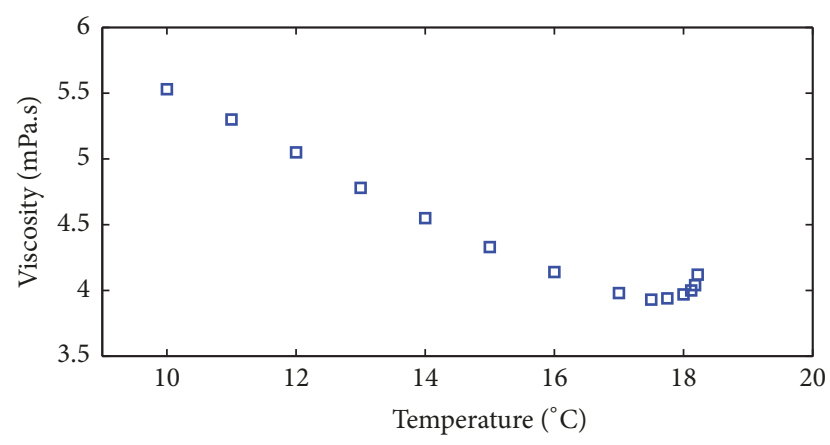

FIGURE 11: Viscosity of the mixture as a function of temperature at the critical composition (32.1\%). The experimental data is obtained from $[21,39]$.

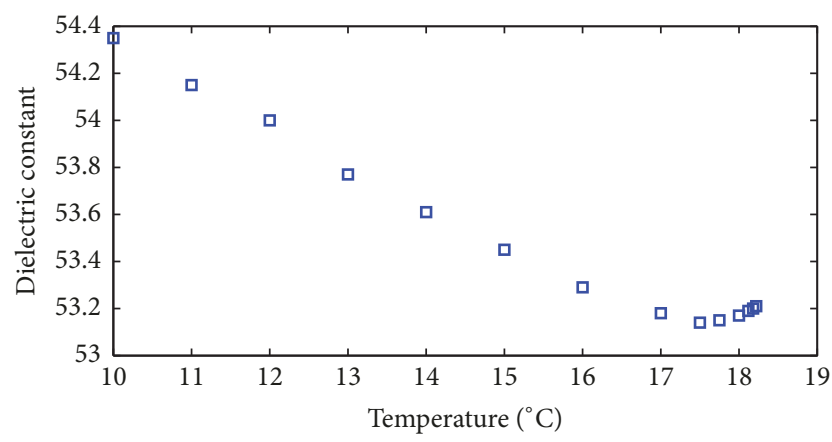

FIgURE 12: Temperature dependence of the dielectric constant of the binary mixture at the critical composition (32.1\%) obtained from [23].

line), the values of the parameters $\mathrm{A}, \mathrm{B}$, and $T_{g}$ at the critical composition are found to be $0.37 \mathrm{mS} / \mathrm{cm}, 102.0 \mathrm{~K}^{-1}$, and $241.0 \mathrm{~K}$, respectively. Therefore, the VFT equation with the obtained fitting parameters is used to relate the electrical conductivity of the binary fluid mixture to temperature in the single phase region $\left(\mathrm{T} \leq \mathrm{T}_{\text {demix }}\right)$.

Temperature dependence of viscosity of the binary mixture for the critical composition in the single phase has been reported by $[21,39]$ as shown in Figure 11. It is shown that when the temperature approaches the critical temperature $\left(\mathrm{T}_{\text {demix }}\right)$, the critical fluctuations cause a further increase of the viscosity for the critical composition (32.1\%).

The dielectric constant as a function of temperature in the single phase region of the binary mixture for the critical composition has been reported by [23]. By extrapolating the experimental data of [23], the temperature dependence of dielectric constant of the mixture at the critical composition is shown in Figure 12. The result shows that the dielectric constant fluctuates near the critical point.

The binary mixtures used in this work may be different from those used in $[21,23,39]$ due to the impurities (e.g., $\mathrm{CO}_{2}$, $\mathrm{H}_{2} \mathrm{O}$ ), the dissolution of mineral substances of the sample, and the ability of the strongly basic triethylamine to attack glass as mentioned in [19]. This is indicated by the shift of $\mathrm{T}_{\text {demix }}$ to higher temperatures at the critical composition of $32.1 \%$. The variations of viscosity and dielectric constant of

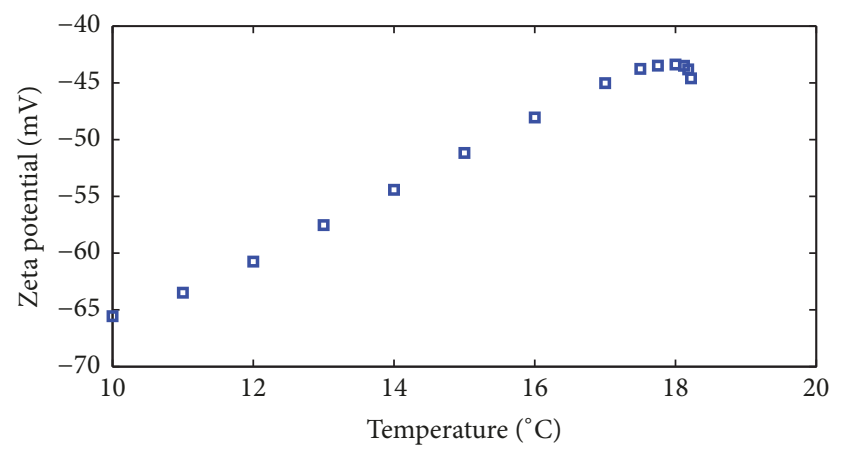

FIGURE 13: The prediction of the zeta potential as a function of temperature at the critical composition for the well defined binary mixture.

the binary mixtures used in our work with temperature, in particular near the demixing phase transition, are not yet measured due to the limitations of experimental condition and equipment. Therefore, we use temperature dependence of viscosity and dielectric constant reported in literature [21, 23, 39 ] on the well defined mixtures to obtain the zeta potential.

To predict how the zeta potential varies near the demixing phase transition point for the well defined mixture at critical composition, we assume that the SPC and the electrical conductivity for the well defined mixture behave similarly to those observed in this work (see Figures 8 and 10). By fitting the experimental data shown in Figures 8 and 10 (see solid lines), the relationship between the SPC and the electrical conductivity against temperature for the critical composition of the well defined mixture is obtained. With the knowledge of viscosity and dielectric constant obtained from $[21,23,39]$, the zeta potential as a function of temperature is obtained using (3) and shown in Figure 13 (because the electrical conductivity of TEA-W mixture is always larger than $0.30 \mathrm{~S} / \mathrm{m}$ as shown in Figure 10, the surface conductance is ignored as reported in [36] for the ceramic artificial rock).

The result predicts that zeta potential at the critical composition (32.1\%) behaves differently around the critical point if the viscosity and dielectric constant follow what has been reported in $[21,23,39]$. To better understand the behavior of the zeta potential, additional studies on the variation of the viscosity and dielectric constant of the own binary mixtures used in this work with temperature need to be performed. It is also seen that the magnitude of the zeta potential decreases with increasing temperature. That is opposite to what has been observed in our earlier work $[26,35,38]$ in which the zeta potential in magnitude increases temperature for aqueous electrolytes. It means that the TEA$\mathrm{W}$ binary mixtures may change the properties of the electric double layer compared to normal aqueous electrolytes.

\section{Conclusions}

The streaming potential measurements for a porous sample saturated with a binary mixture of triethylamine-water with three different mass fractions have been carried out. From the measured SPC, the variation of the zeta potential with 
temperature in single phase solution is predicted for the critical composition. The results show that there is no anomaly in the SPC as the temperature approaches the demixing temperatures for all three mass fractions of TEA. It is also seen that the streaming potential coefficient and the zeta potential in magnitude decrease with increasing temperature. This observation is opposite to what has been observed in literature for aqueous solutions. It means that the properties of the electric double layer for the TEA-W mixtures are different from those for aqueous electrolytes. The zeta potential for the critical composition is predicted to fluctuate around the critical point. To verify the behavior of the SPC and the zeta potential near the critical phase transition, the viscosity and dielectric constant of the own binary mixtures used in this work need to be measured as a function of temperature. In addition to the measurements of the viscosity and dielectric constant, the TEA-W mixtures need to be degassed by the Freeze-Pump-Thaw technique [40] and protected from the atmosphere. To remove the effect of temperature, few more different ionic strengths of electrolyte mixed with TEA may be performed in our proposed future work. This work, along with the conductivity and viscosity measurements from $[21,22]$, the dielectric constant measurement from [23], the refractive index measurement from [41], and the mass density measurement from [42], creates a better understanding of the critical behavior of TEA-W binary mixtures near the demixing phase transition.

\section{Data Availability}

The data used to support the findings of this study are included within the article.

\section{Conflicts of Interest}

The authors declare that there are no conflicts of interest regarding the publication of this paper.

\section{Acknowledgments}

The first author would like to thank the Vietnam National Foundation for Science and Technology Development (NAFOSTED) for financial support under grant number 103.99-2016.29.

\section{References}

[1] B. Wurmstich and F. D. Morgan, "Modeling of streaming potential responses caused by oil well pumping," Geophysics, vol. 59, no. 1, pp. 46-56, 1994.

[2] T. Ishido and J. W. Pritchett, "Numerical simulation of electrokinetic potentials associated with subsurface fluid flow," Journal of Geophysical Research: Solid Earth, vol. 104, no. 7, pp. 1524715259, 1999.

[3] R. F. Corwin and D. B. Hoover, "The self-potential method in geothermal exploration," Geophysics, vol. 44, no. 2, pp. 226-245, 1979.

[4] G. Saracco, P. Labazuy, and F. Moreau, "Localization of self-potential sources in volcano-electric effect with complex continuous wavelet transform and electrical tomography methods for an active volcano," Geophysical Research Letters, vol. 31, no. 12, 2004.

[5] G. Mauri, G. Williams-Jones, and G. Saracco, "Depth determinations of shallow hydrothermal systems by self-potential and multi-scale wavelet tomography," Journal of Volcanology and Geothermal Research, vol. 191, no. 3-4, pp. 233-244, 2010.

[6] J. H. Saunders, M. D. Jackson, and C. C. Pain, "Fluid flow monitoring in oil fields using downhole measurements of electrokinetic potential," Geophysics, vol. 73, no. 5, pp. E165E180, 2008.

[7] H. Mizutani, T. Ishido, T. Yokokura, and S. Ohnishi, "Electrokinetic phenomena associated with earthquakes," Geophysical Research Letters, vol. 3, no. 7, pp. 365-368, 1976.

[8] J. Pozzi and L. Jouniaux, "Electrical effects of fluid circulation in sediments and seismic prediction," Comptes rendus de l'Académie des Sciences - Serie II, vol. 318, no. 1, pp. 73-77, 1994.

[9] L. Jouniaux and J. P. Pozzi, "Streaming potential and permeability of saturated sandstones under triaxial stress: consequences for electrotelluric anomalies prior to earthquakes," Journal of Geophysical Research, vol. 100, no. B6, pp. 10197-10209, 1995.

[10] P. M. Reppert and F. D. Morgan, "Temperature-dependent streaming potentials: 2. Laboratory," Journal of Geophysical Research, vol. 108, no. B11, p. 2547, 2003.

[11] A. Bolève, A. Crespy, A. Revil, F. Janod, and J. L. Mattiuzzo, "Streaming potentials of granular media: influence of the Dukhin and Reynolds numbers," Journal of Geophysical Research, vol. 112, no. B8, Article ID B08204, 2007.

[12] J. Vinogradov, M. Z. Jaafar, and M. D. Jackson, "Measurement of streaming potential coupling coefficient in sandstones saturated with natural and artificial brines at high salinity," Journal of Geophysical Research, vol. 115, no. B12, 2010.

[13] P. W. J. Glover and N. Déry, "Streaming potential coupling coefficient of quartz glass bead packs: dependence on grain diameter, pore size, and pore throat radius," Geophysics, vol. 75, no. 6, pp. F225-F241, 2010.

[14] R. Hidalgo-Alvarez, A. Delgado, J. Callejas, and F. GonzálezCaballero, "Electrophoretic behaviour of calcium oxalate monohydrate in liquid mixtures," Colloid and Polymer Science, vol. 263, no. 11, pp. 941-947, 1985.

[15] F. J. R. Hernandez, "Dependence of the electrophoretic coefficient of polystyrene model colloids on the solvent composition," Journal of Colloid and Interface Science, vol. 203, no. 1, pp. 208213, 1998.

[16] R. Hidalgo-Alvarez, F. J. de las Nieves, and G. Pardo, "Concentration dependence of electrokinetic transport coefficients of non-aqueous binary mixtures through weakly charged porous plugs," Journal of the Chemical Society, Faraday Transactions 1: Physical Chemistry in Condensed Phases, vol. 81, no. 3, pp. 609619, 1985.

[17] M. Srivastava and S. N. Lal, "Electro-kinetic transport of acetone, water and their binary mixtures," Colloid and Polymer Science, vol. 258, no. 7, pp. 887-882, 1980.

[18] U. Ghosh, T. L. Borgne, D. Jougnot, N. Linde, and Y. Méheust, "Geoelectrical signatures of reactive mixing: a theoretical assessment," Geophysical Research Letters, vol. 45, no. 8, pp. 3489-3498, 2018.

[19] C. Stenland and B. M. Pettitt, "Binary-solution critical opalescence: mole fraction versus temperature phase diagram," Journal of Chemical Education, vol. 72, no. 6, pp. 560-564, 1995.

[20] E. S. Gopal, “Critical opalescence," Resonance, vol. 5, no. 4, pp. 37-45, 2000. 
[21] N. Hafaiedh, A. Toumi, and M. Bouanz, "Dynamic viscosity study of binary mixtures triethylamine + water at temperatures ranging from (283.15 to 291.35) K," Journal of Chemical \& Engineering Data, vol. 54, no. 8, pp. 2195-2199, 2009.

[22] A. Toumi, N. Hafaiedh, and M. Bouanz, "Electrical conductivity investigation of diluted potassium chloride solutions in binary mixture triethylamine-water near its consolute point," Fluid Phase Equilibria, vol. 305, no. 2, pp. 185-191, 2011.

[23] J. Hamelin, T. K. Bose, and J. Thoen, "Critical behavior of the dielectric constant in the triethylamine-water binary liquid mixture: evidence of an intrinsic effect," Physical Review E: Statistical, Nonlinear, and Soft Matter Physics, vol. 53, no. 1, pp. 779-784, 1996.

[24] P. Atkins and J. De Paula, Atkins' Physical Chemistry, Oxford University Press, 2014.

[25] O. Stern, "Zur Theorie der elektrolytischen Doppelschicht," Zeitschrift für Elektrochemie, vol. 30, no. 21-22, pp. 508-516, 1924.

[26] T. Ishido and H. Mizutani, "Experimental and theoretical basis of electrokinetic phenomena in rock-water systems and its applications to geophysics," Journal of Geophysical Research, vol. 86, no. B3, pp. 1763-1775, 1981.

[27] F. D. Morgan, E. R. Williams, and T. R. Madden, "Streaming potential properties of westerly granite with applications," Journal of Geophysical Research, vol. 94, no. B9, pp. 12449-12461, 1989.

[28] H. M. Jacob and B. Subirm, Electrokinetic and Colloid Transport Phenomena, Wiley-Interscience, 2006.

[29] R. J. Hunter, Zeta Potential in Colloid Science, Academic, New York, NY, USA, 1981.

[30] J. Davis, R. James, and J. Leckie, "Surface ionization and complexation at the oxide/water interface: I. Computation of electrical double layer properties in simple electrolytes," Journal of Colloid and Interface Science, vol. 63, no. 3, pp. 480-499, 1978.

[31] B. Nourbehecht, Irreversible thermodynamic effects in inhomogeneous media and their applications in certain geoelectric problems [Ph.D. thesis], MIT Press, Cambridge, Mass, USA, 1963.

[32] L. Jouniaux and T. Ishido, "Electrokinetics in earth sciences: a tutorial," International Journal of Geophysics, vol. 2012, Article ID 286107, 16 pages, 2012.

[33] L. Jouniaux, M. L. Bernard, M. Zamora, and J. P. Pozzi, "Streaming potential in volcanic rocks from Mount Pelée," Journal of Geophysical Research: Solid Earth, vol. 105, no. B4, pp. 8391-8401, 2000.

[34] L. D. Thanh, P. Van Do, N. Van Nghia, and N. X. Ca, "A fractal model for streaming potential coefficient in porous media," Geophysical Prospecting, vol. 66, no. 4, pp. 753-766, 2018.

[35] D. T. Luong and R. Sprik, "Examination of a theoretical model of streaming potential coupling coefficient," International Journal of Geophysics, vol. 2014, Article ID 471819, 12 pages, 2014.

[36] L. D. Thanh and R. Sprik, "Permeability dependence of streaming potential coefficient in porous media," Geophysical Prospecting, vol. 64, no. 3, pp. 714-725, 2016.

[37] D. T. Luong, Electrokinetics in porous media [Ph.D. thesis], University of Amsterdam, Netherlands, 2014.

[38] D. B. Pengra, S. X. Li, and P.-Z. Wong, "Determination of rock properties by low-frequency AC electrokinetics," Journal of Geophysical Research: Solid Earth, vol. 104, no. 12, pp. 2948529508, 1999.
[39] A. Toumi, N. Hafaiedh, and M. Bouanz, "Study of the transport properties of the critical binary mixture triethylamine - water with an ionic impurity," Ukrainian Journal of Physics, vol. 56, no. 8, pp. 816-823, 2011.

[40] F. Kohler and O. K. Rice, "Coexistence curve of the triethylamine-water system," Journal of Chemical Physics, vol. 26, no. 6, pp. 1614-1618, 1957.

[41] C. Pépin, T. K. Bose, and J. Thoen, "Evidence for an intrinsic refractive-index anomaly in a critical binary liquid mixture," Physical Review Letters, vol. 60, no. 24, pp. 2507-2510, 1988.

[42] G. P. Furrow and S. C. Greer, "Critical behavior of the density, heat capacity, and refractive index for triethylamine + water," The Journal of Chemical Physics, vol. 79, no. 7, pp. 3474-3481, 1983. 

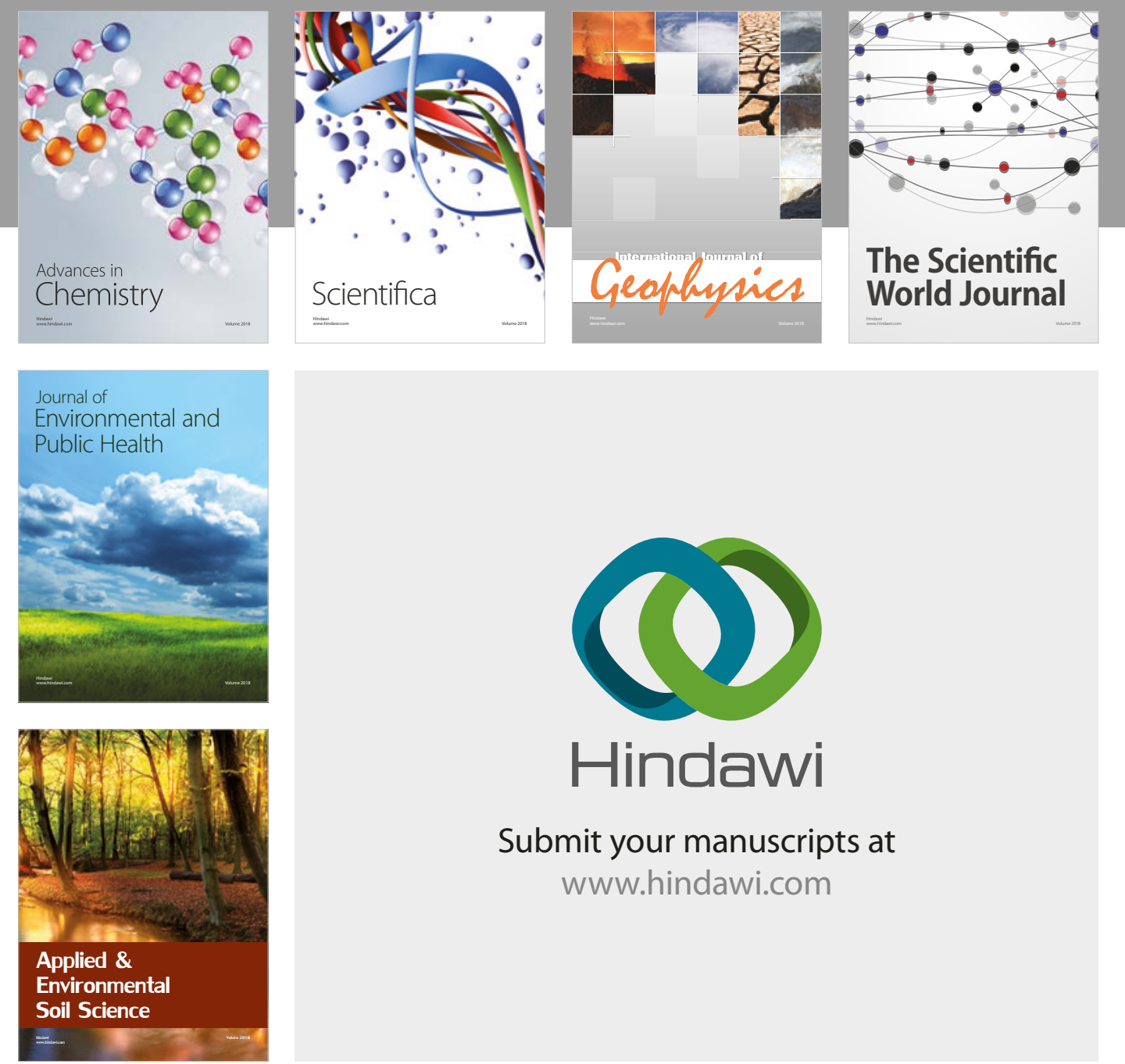

The Scientific

\section{World Journal}
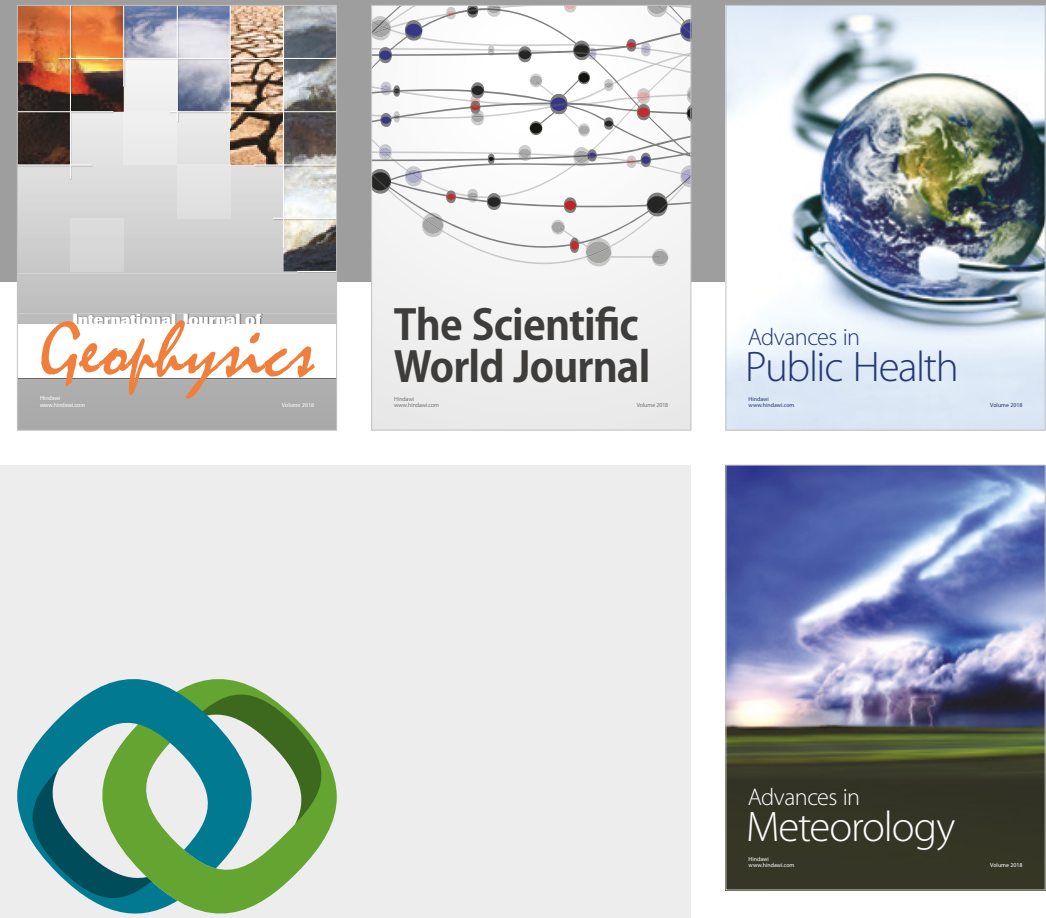

Advan

Public Health

\section{Hindawi}

Submit your manuscripts at

www.hindawi.com
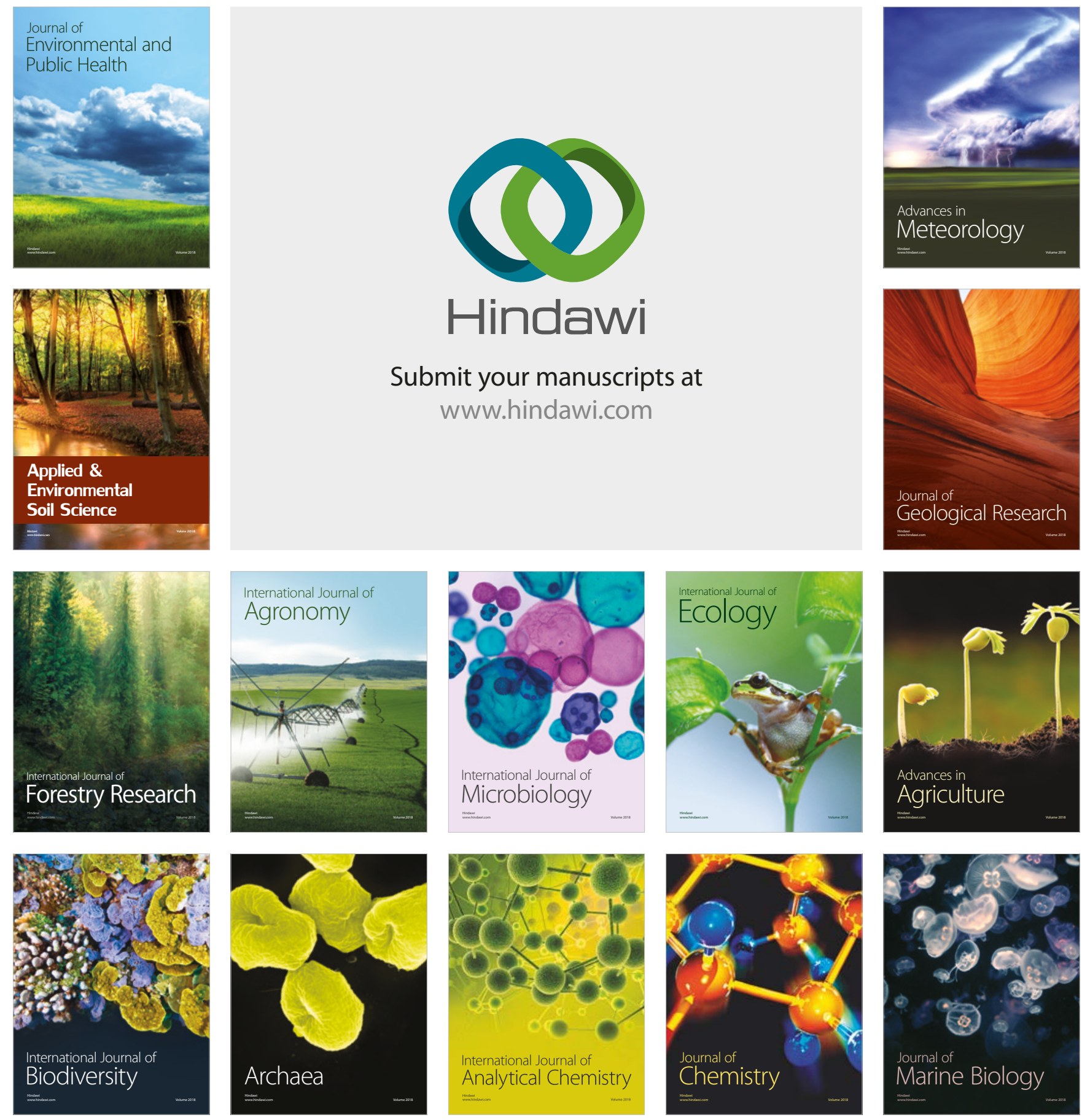